\title{
Humedales del Parque Nacional Chirripó, Costa Rica: características, relaciones geomorfológicas y escenarios de cambio climático
}

\author{
Néstor Veas-Ayala ${ }^{1}$, Adolfo Quesada-Román ${ }^{2,3}$ Hugo G. Hidalgo ${ }^{4,5}$ \& Eric J. Alfaro ${ }^{4,5,6}$ \\ 1.Proyecto Humedales, PNUD-SINAC-GEF. Santo Domingo de Heredia; nestor.veas@undp.org, torneca@gmail.com \\ 2. Climate Change Impacts and Risks in the Anthropocene (C-CIA), Institute for Environmental Sciences, University of \\ Geneva, Switzerland; adolfo.quesada@unige.ch \\ 3. Escuela de Geografía, Universidad de Costa Rica, San Pedro 2060, Costa Rica; adolfo.quesadaroman@ucrac.cr \\ 4. Escuela de Física, Universidad de Costa Rica, San Pedro 2060, Costa Rica; hugo.hidalgo@ucr.ac.cr, \\ erick.alfaro@ucr.ac.cr \\ 5. Centro de Investigaciones Geofísicas, Universidad de Costa Rica, San Pedro 2060, Costa Rica; \\ hugo.hidalgo@ucr.ac.cr, erick.alfaro@ucr.ac.cr \\ 6. Centro de Investigación en Ciencias del Mar y Limnología, Universidad de Costa Rica, San Pedro 2060, Costa Rica; \\ erick.alfaro@ucr.ac.cr
}

Recibido 16-IV-2018. Corregido 13-VII-2018. Aceptado 17-VIII-2018.

\begin{abstract}
Wetlands of the Chirripó National Park, Costa Rica: characteristics, geomorphological relationships and climate change scenarios. The geomorphological, climatological and hydrological characteristics of the high Andean wetlands of the Chirripó National Park (PNCh) are analyzed. The PNCh is located in south-central Costa Rica, Central America. This analysis includes a geomorphological survey on a 1: 25000 scale of the relief forms and processes, particularly those of glacial origin. The National Wetland Inventory carried out by the Wetlands Project (UNDP-SINAC-GEF) was also used, along with different climate change projections corresponding to the scenarios for the 2039-2059 and 2079-2099 horizons. The main objective is to characterize the current state of these ecosystems, to explain how and why they are formed, as well as to assess the possible implications in the hydrological cycle and the water supply of these wetlands due to future climate variations. The results showed the relevance of these natural reservoirs in maintaining the ecological dynamics of the PNCh. We also studied the services of water provision and regulation of the hydrological cycle in the basins that compose this protected area.
\end{abstract}

Key words: wetlands; geomorphology; climatology; hydrology; climate change; Central America.

Veas-Ayala, N., Quesada-Román, A., Hidalgo, H. G. \& Alfaro, E. J. (2018). Humedales del Parque Nacional Chirripó, Costa Rica: características, relaciones geomorfológicas y escenarios de cambio climático. Revista de Biología Tropical, 66(4), 1436-1448.

Los humedales se definen como las extensiones de marismas, pantanos y turberas, o superficies cubiertas de aguas, sean éstas de régimen natural o artificial, permanentes o temporales, estancadas o corrientes, dulces, salobres o saladas, incluidas en las extensiones de agua marina cuya profundidad en marea baja no exceda los seis metros (Secretaría de la Convención Ramsar, 2013). En las latitudes tropicales, si bien la mayor superficie de humedales se concentra en altitudes bajas y medias, los humedales de altura tienen una gran relevancia por su función dentro del ciclo hidrológico, así como reguladores climáticos y fijación de carbono, entre otros (Millenium Assessment, 2005). Estos humedales, también llamados altoandinos, han sido poco estudiados y caracterizados en Costa Rica, y son 
representados por lagos, lagunas estacionales, pantanos y turberas de montaña, considerados por la Convención Ramsar como de gran fragilidad, asociada a causas como el cambio climático, y tienen un origen glaciar, volcánico y tectónico (WWF Colombia, 2005).

Dadas sus extensiones, los humedales más estudiados en Costa Rica han sido los manglares; de manera contraria, los menos investigados han sido los de altura, en especial por la dificultad de acceso a los mismos y el poco conocimiento general que se ha generado a partir de ellos (Veas-Ayala, 2015). Estos humedales se concentran en la Cordillera de Talamanca, donde se encuentran las mayores altitudes del país, y se reconoce la existencia de lagos y lagunas permanentes como estacionales en sus alrededores (Horn, Orvis, \& Haberyan, 2005). El objetivo de este trabajo es presentar los resultados del inventario de los humedales del Parque Nacional Chirripó (PNCh), asociarlos con sus características geomorfológicas y los escenarios de cambios en el clima de los periodos 2039-2059 у 2079-2099, para analizar las posibles afectaciones en la dinámica natural de las formas de relieve glaciar, así como los humedales.

\section{MATERIALES Y MÉTODOS}

Descripción de sitio: El PNCh se localiza en las coordenadas extremas de $9^{\circ} 22^{\prime} 43.824^{\prime \prime}$ - 9॰36'45.706" N \& 83²5'5.915” 8339'49.048" W (Fig. 1). Hace 5 Ma el territorio costarricense y panameño estaba formado por un rosario de islas dispuestas en un arco volcánico. En este periodo se dio la colisión de la Serranía de Cocos con la Placa Caribe; este fenómeno puso fin, al vulcanismo de la Cordillera de Talamanca hace 2 Ma (Morell, 2015).

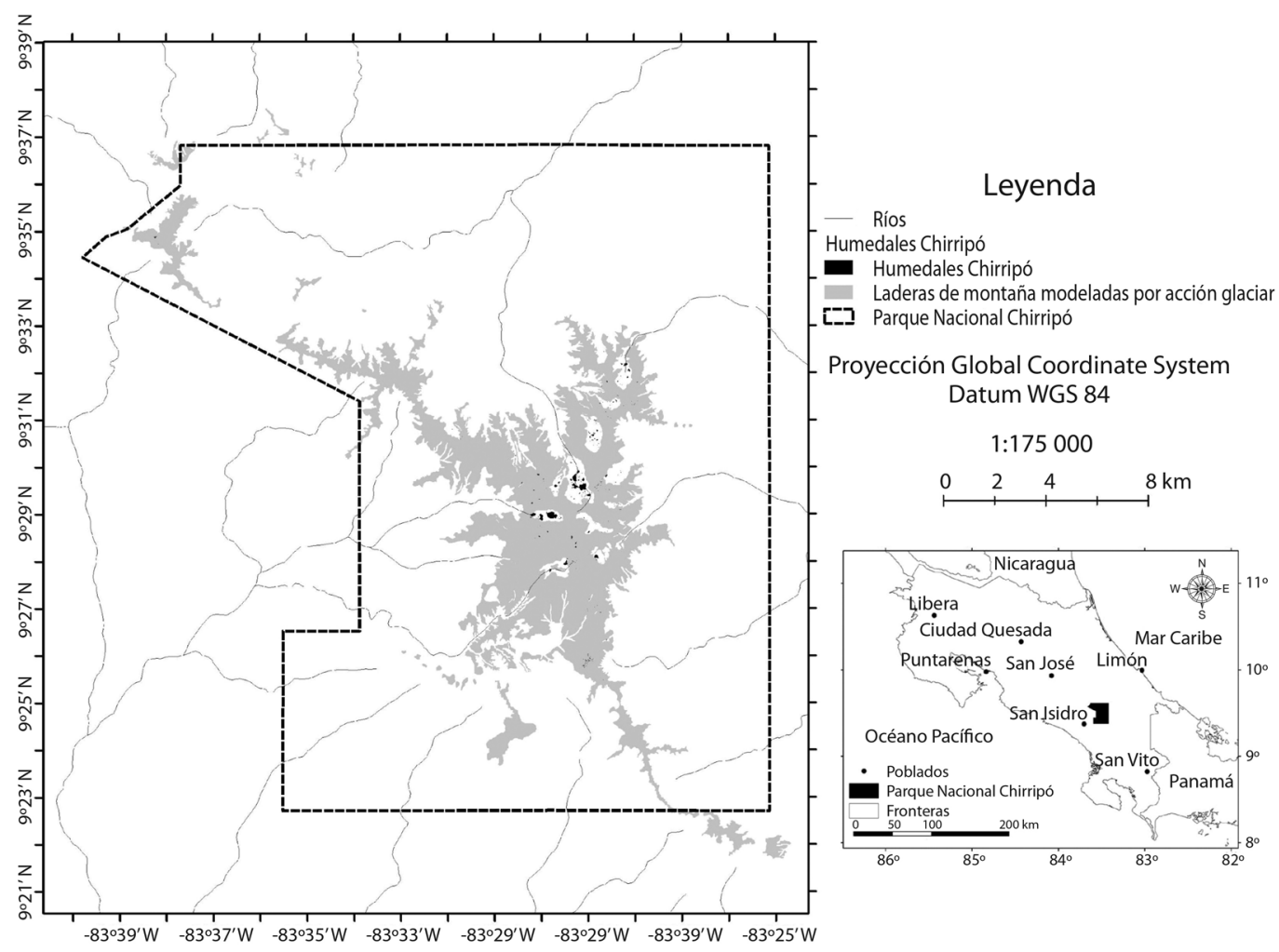

Fig. 1. Localización a escala nacional y regional del PNCh, así como sus humedales.

Fig. 1. Localization at national and regional level of the PNCh, as well as its wetlands. 
Su geología se constituye por rocas plutónicas (granodioritas) del Mioceno y volcánicas (andesitas) de edad Mio-Plioceno (Denyer \& Alvarado, 2007).

Los efectos del Último Máximo Glaciar (UMG) en las cumbres de la Cordillera de Talamanca y el PNCh permitieron el desarrollo de áreas cubiertas de glaciares y nieves que favorecieron el modelado de formas glaciares tanto erosivas como acumulativas que en la actualidad han dejado relictos de esta dinámica (Horn, 1990; Orvis \& Horn, 2000, 2005; Lachniet \& Seltzer, 2002). Entre hace 20 mil años (20 ka) y el establecimiento del clima actual (8 $\mathrm{ka}$ ), se dio una transición paulatina expresada en un aumento de las precipitaciones y temperaturas a escala regional y local (Islebe \& Hooghiemstra, 1997). Con el retroceso y desaparición de los glaciares, inicia el desarrollo de los procesos paraglaciales, donde se intensifican las tasas de erosión debido al aporte de sedimentos dejados por la dinámica glaciar, y aumenta la incidencia de procesos de ladera (Ballantyne, 2002).

De acuerdo al IMN (2008) la temperatura promedio en la zona de estudio es de 8 ${ }^{\circ} \mathrm{C}$, mientras que la precipitación se mantiene en un rango que va de los 2000 a $5500 \mathrm{~mm}$ (promedio anual). La estación Chirripó (3630 $\mathrm{msnm}$ ) ha reportado una precipitación menor a $100 \mathrm{~mm}$ mensuales durante diciembre y abril; no obstante, entre mayo y noviembre las lluvias sobrepasan los $150 \mathrm{~mm}$ mensuales (IMN, 2009). El climograma presenta dos picos pluviométricos (Cuadro 1), el primero es de 300 $\mathrm{mm}$ en mayo y tiene relación con el inicio de la temporada de lluvias. También muestra un descenso durante junio y agosto, como respuesta al veranillo (Maldonado, Rutgersson, Alfaro, Amador, \& Claremar, 2016).

El PNCh tiene una importancia hidrológica trascendente a nivel regional ya que allí nacen los ríos Chirripó, Telire, Reventazón y el Pacuare que desembocan en el Mar Caribe. En el Pacífico surgen los cauces del Savegre y el General; este último integra la cuenca alta del río Grande de Térraba al sumar la cuenca del río Coto Brus (Quesada-Román, 2017). Asimismo, una gran cantidad de nacientes en ambas vertientes se alimentan de las aguas subterráneas que provienen del Parque.

Los ecosistemas presentes en el PNCh están definidos por cuatro pisos altitudinales dentro de sus límites: subalpino, montano, montano bajo y premontano (Chaverri, 2008), los más representativos por superficie e importancia son el montano y el subalpino. El piso subalpino del PNCh representa la mayor superficie de páramo de Costa Rica, y comprende la unidad paisajística que está por encima de la línea continua de vegetación arbórea y hasta los $3820 \mathrm{msnm}$ del Cerro Chirripó

CUADRO 1

Variables ambientes de la estación meteorológica Chirripó del IMN, 1995-2009

TABLE 1

Environmental variables from the IMN's Chirripó meteorological station, 1995-2009

\begin{tabular}{lccccccccccccc}
\multicolumn{1}{c}{ Elementos } & Ene & Feb & Mar & Abr & May & Jun & Jul & Ago & Set & Oct & Nov & Dic & Anual \\
Lluvia $(\mathrm{mm})$ & 56.2 & 20.8 & 40.0 & 84.0 & 304.2 & 204.8 & 148.3 & 180.3 & 302.3 & 314.7 & 231.9 & 87.1 & 1974.6 \\
Días con lluvia & 18 & 11 & 13 & 17 & 27 & 26 & 27 & 27 & 28 & 28 & 26 & 21 & 269 \\
Temp. Máx. $\left({ }^{\circ} \mathrm{C}\right)$ & 15.4 & 16.4 & 16.0 & 15.5 & 14.6 & 14.3 & 14.2 & 14.1 & 14.0 & 13.7 & 14.0 & 14.9 & 14.8 \\
Temp. Mín. $\left({ }^{\circ} \mathrm{C}\right)$ & 3.3 & 3.6 & 4.3 & 4.8 & 5.4 & 5.0 & 4.5 & 4.7 & 4.8 & 5.0 & 4.7 & 3.9 & 4.5 \\
Temp. Med. $\left({ }^{\circ} \mathrm{C}\right)$ & 9.4 & 10.0 & 10.2 & 10.2 & 10.0 & 9.7 & 9.4 & 9.4 & 9.4 & 9.4 & 9.4 & 9.4 & 9.6 \\
Viento $(\mathrm{km} / \mathrm{h})$ & 31.5 & 25.6 & 19.3 & 18.9 & 16.9 & 23.1 & 29.4 & 27.8 & 17.5 & 16.4 & 25.1 & 32.4 & 23.7 \\
Humedad $(\%)$ & 72 & 62 & 67 & 77 & 89 & 91 & 91 & 92 & 92 & 93 & 90 & 79 & 83 \\
\hline
\end{tabular}

Fuente: Instituto Meteorológico Nacional de Costa Rica (2009).

Source: National Institute of Meteorology of Costa Rica (2009). 


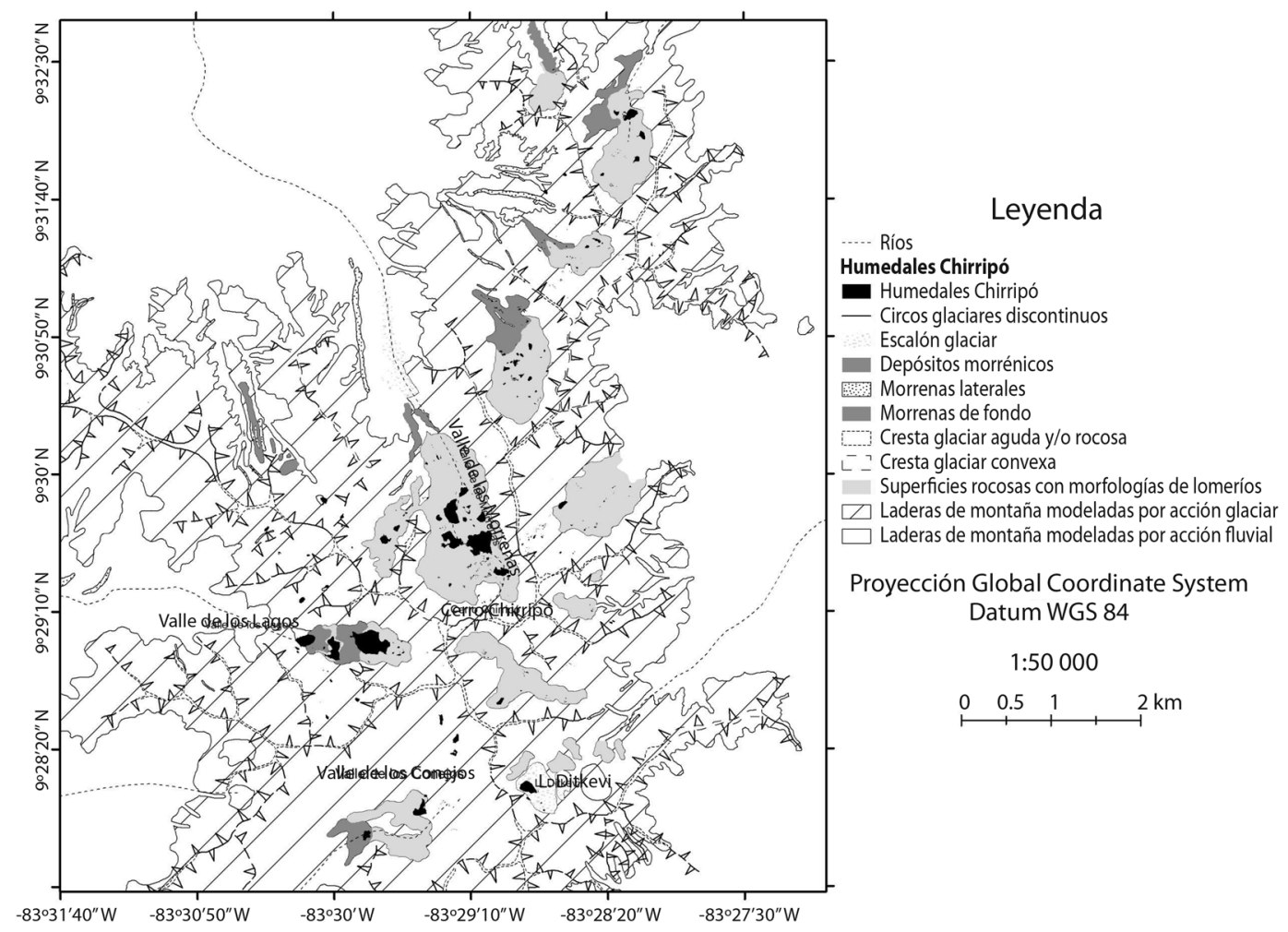

Fig. 2. Geomorfología glaciar de la región de mayor densidad de humedales del PNCh.

Fig. 2. Glacial geomorphology of the major density region of wetlands of the PNCh.

(Horn et al., 2005; Horn \& Haberyan, 2016; Kappelle \& Horn, 2016). Los suelos son relativamente jóvenes y poco desarrollados; se han identificado andisoles, inceptisoles, histosoles y entisoles (Kapelle \& Uffelen, 2005). Este hecho, aunado a los extremos climáticos de este ecosistema, favorecen que exista un $60 \%$ de flora vascular endémica (Kapelle, 2005), así como un predominio del bambú enano, o chusquea (Cleef \& Chaverri, 1992).

\section{Cartografía y caracterización geomor-}

fológica: Se interpretaron fotografías aéreas a escala 1:25 000 (PRIAS-CENAT, 2005). Los métodos gráficos usados provienen de Gustavsson, Kolstrup y Seijmonsbergen (2006) y Quesada-Román (2016). Se obtuvo un mapa preliminar a escala 1:25 000 que muestra las diferentes formas de relieve de origen glaciar y su asociación con los humedales presentes en el PNCh (Fig. 2).

Mapeo, clasificación y caracterización de humedales: Para la cartografía de humedales en el PNCh se realizó una gira de campo de 12 días en marzo 2016, visitando los sectores de Cerro Amó, Valle de las Morrenas, Fila Norte, además de los senderos a las lagunas San Juan y Ditkevi. Se georreferenció con GPS cada uno de los humedales encontrados, además de ser marcados con una cinta para no repetir el ejercicio. Cada una de estas unidades fue caracterizada en una base de datos adjunta a la capa, donde se incluyó el tipo y subtipo de humedal según el Manual para la identificación y clasificación de humedales en Costa Rica (Bravo \& Windevoxhel, 1997) y el Decreto 35803-MINAET (Decreto Ejecutivo 35803, 2010). 
La digitalización se realizó sobre las fotografías aéreas del Proyecto BID-Catastro (2005), las cuales se encuentran a escala 1:5 000, de allí que esta misma relación es la que se mantuvo en el mapeo resultante. Si bien las imágenes tienen más de 10 años, al ser el PNCh una categoría de manejo que no permite alteraciones al ecosistema, y tal como fue corroborado en el campo, los humedales mantienen tanto su ubicación como su área, siendo este mapeo válido hasta fechas presentes.

Escenarios de cambio climático: La línea base del clima fue establecida en el período 1979-1999 y los escenarios en el clima fueron estimados para su análisis de manera anual ya que existen pocas diferencias en los cambios de las estacionalidades de las variables. A partir de la línea base climática generada con la información entre 1979 y 1999, así como los escenarios de cambios en el clima de los periodos 2039-2059 y 2079-2099 en la región del PNCh, se analizan las posibles afectaciones en la dinámica natural de las formas de relieve glaciar, así como los humedales.

Se usaron los datos de 7 modelos de CMIP5 (Coupled Model Intercomparison Project versión 5) listados en el cuadro 2, para los escenarios de concentraciones rcp4.5 y rep8.5. Para el caso de rep8.5 se dispuso de las 14 corridas mostradas en el cuadro 2, mientras que para el rcp4.5 se dispuso de 13 corridas, correspondientes a todas las mostradas en el cuadro 2 con excepción del modelo cesm1 cam5m, corrida 3. Los datos de los modelos globales fueron cambiados de escala usando el método estadístico descrito en Hidalgo, Alfaro y Quesada-Montano (2017) para convertirlos en estimados a $5 \times 5 \mathrm{~km}$ de resolución. Se seleccionaron estos modelos debido a su capacidad para reproducir aspectos importantes del clima en América Central (Hidalgo \& Alfaro, 2015). Esto se hizo a nivel regional. A nivel local del PNCh no se dispone de información meteorológica suficiente para validar a una escala adecuada las simulaciones durante el período histórico utilizado. Los datos de precipitación y temperatura fueron usados para calcular los impactos en estas variables debido al cambio climático para los dos horizontes mencionados en el párrafo anterior. También se muestran la evolución temporal de la precipitación y temperatura agregada para todo el PNCh. Debido a que los cambios para el escenario rcp4.5 son muy pequeños, no se mostraron los mapas correspondientes, solo su evolución temporal (Fig. 3 y Fig. 4).

\section{RESULTADOS}

Geomorfología de las áreas cubiertas por humedales del PN Chirripó: Entre las formas de origen glaciar de carácter erosivo

CUADRO 2

Modelos de circulación usados en este estudio

TABLE 2

Circulation models used in this study

\begin{tabular}{lllc}
$\#$ & \multicolumn{1}{c}{ Modelo } & \multicolumn{1}{c}{ Centro o grupo de modelado } & $\begin{array}{c}\text { Número } \\
\text { de corridas }\end{array}$ \\
1 & ccsm4 & Canadian Centre for Climate Modelling and Analysis (CCCMA) & 3 \\
2 & cesm1_cam5 & Centro Euro-Mediterraneo per I Cambiamenti Climatici (CMCC) & 3 \\
3 & cmec_cms & CMCC & 1 \\
4 & ec_earth & EC-EARTH consortium & 1 \\
5 & giss_e2_r & Geophysical Fluid Dynamics Laboratory (GFDL) & 1 \\
6 & miroc5 & Atmosphere and Ocean Research Institute (The University of Tokyo), National & 2 \\
& & Institute for Environmental Studies, and Japan Agency for Marine-Earth Science and & 3 \\
& & Technology. (MIROC) & \\
7 & mpi_esm_lr & Max Planck Institute for Meteorology (MPI-M) & 3 \\
\hline
\end{tabular}



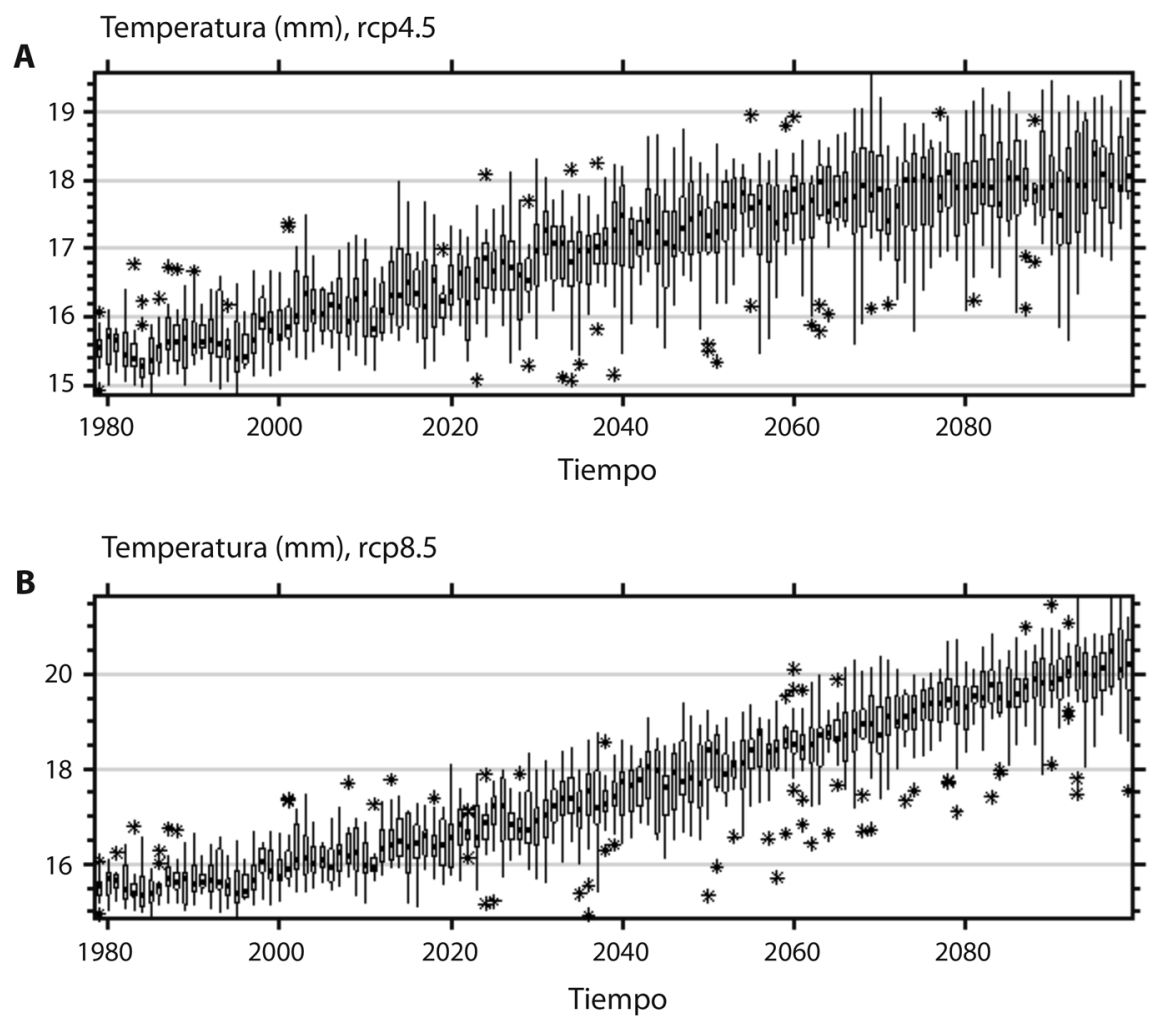

Fig. 3. Proyecciones de temperatura anual $\left({ }^{\circ} \mathrm{C}\right)$ 1979-2099 para las corridas analizadas en la región del PNCh. Las cajas muestran el rango intercuartil y la mediana. Las líneas verticales muestran 1.5 veces el rango intercuartil o la extensión de los datos. Valores fuera de las líneas verticales se muestran como valores atípicos con el símbolo asterisco.

Fig. 3. Projections of annual temperature $\left({ }^{\circ} \mathrm{C}\right)$ 1979-2099 for the runs analyzed in the PNCh region. The box-plots show the interquartile range and the median. The whiskers extend to 1.5 the interquartile range or to the data extend. Values outside the whiskers (outliers) are shown with the asterisk symbol.

se consideran los circos glaciares discontinuos, escalón glaciar, depósitos morrénicos, morrenas de fondo, morrenas laterales, cresta glaciar aguda y/o rocosa, cresta glaciar convexa, superficies rocosas con morfologías de lomeríos, y laderas de montaña modeladas por acción glaciar. Las laderas de montaña modeladas por la acción fluvial sirven de enlace, entre la cumbre y los terrenos bajos ubicados en la cercanía de los lechos fluviales. Este tipo de vertientes se caracterizan por ser heterogéneas en cuanto a litología, longitud, orientación y morfología de laderas. Estas formas de relieve se asocian con relieves fluviales y gravitacionales. El relieve fluvial consta de valles, circos, cabeceras, barrancos y cárcavas mientras que, por otro lado, el relieve gravitacional se explica con geoformas vinculadas con procesos de ladera (Fig. 2).

Identificación, clasificación y características de los humedales del PNCh: El mapeo realizado indica que el $\mathrm{PNCh}$ posee 963 unidades de humedales de dos tipos: lacustre y palustre. En el primer caso, se contabilizaron 9, cubriendo un área total de 27.23 ha, siendo la más grande la Morrena Central, seguida de la Laguna San Juan, con 8.3 y 7.69 ha respectivamente. Los palustres en cambio, totalizaron 954 unidades, abarcando 20.22 ha (Proyecto Humedales, s.f.).

Los lagos y lagunas almacenan agua de manera permanente, regulando y aportando a los flujos hídricos aguas abajo; lo anterior es 


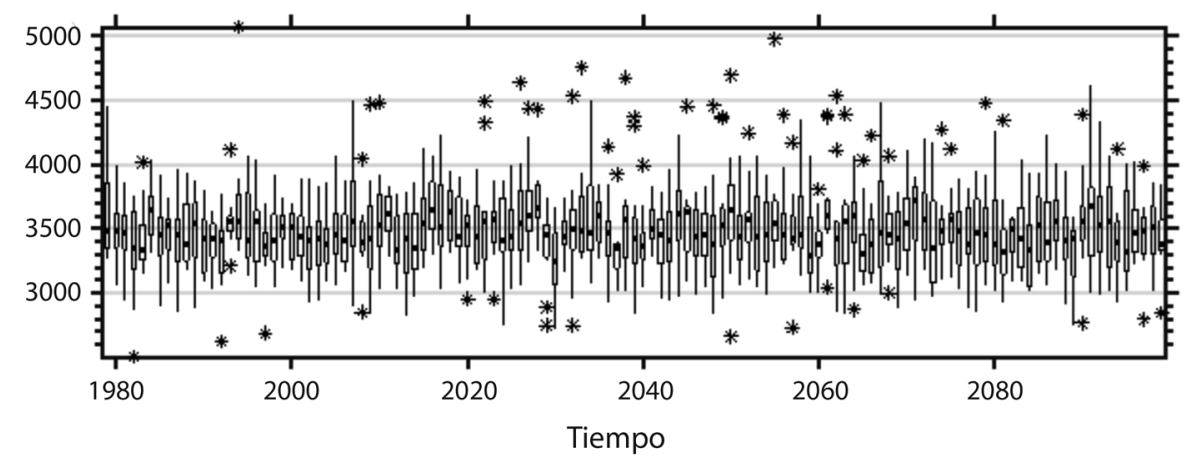

B Precipitación (mm), rcp8.5

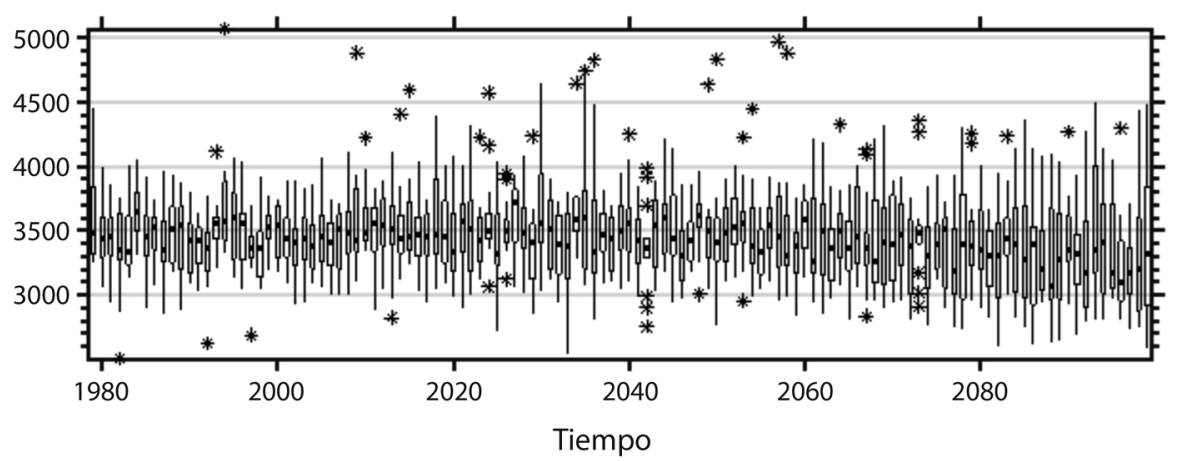

Fig. 4. Proyecciones de precipitación anual (mm) 1979-2099 para las corridas analizadas en la región del PNCh. Las cajas muestran el rango intercuartil y la mediana. Las líneas verticales muestran 1.5 veces el rango intercuartil o la extensión de los datos. Valores fuera de las líneas verticales se muestran como valores atípicos con el símbolo asterisco.

Fig. 4. Projections of annual precipitation (mm) 1979-2099 for the runs analyzed in the PNCh region. The box-plots show the interquartile range and the median. The whiskers extend to 1.5 the interquartile range or to data extenda. Values outside the whiskers (outliers) are shown with the asterisk symbol.

posible gracias al represamiento natural causado por las morrenas, quienes impiden el normal movimiento de las aguas y acumulan el líquido en depresiones y sitios relativamente planos, formados por los relictos glaciares. Sus espejos de agua son igualmente importantes para las aves migratorias y especies de fauna que toman agua de ellas.

Las lagunas estacionales, clasificadas como palustres al no poseer un espejo de agua permanente y más bien tener un flujo de agua intermitente, tienen un aporte de agua proveniente de las precipitaciones, la escorrentía producto de las mismas lluvias y la condensación del vapor de agua. El veloz secado de las mismas obedece a la fuerte radiación solar, la baja cobertura vegetal y la infiltración.

Estos humedales se presentan especialmente en sectores de pendiente más pronunciadas dentro del PNCh, dando como resultado que tengan superficies pequeñas, pero con una amplia distribución, ubicándose en prácticamente todo el páramo del parque, incluso en zonas de pendiente, especialmente en los sectores de Cerro Amó, Laguna Ditkevi, Fila Norte, las Morrenas y Urán.

Escenarios de cambio climático para el PNCh: La figura 3 muestra las proyecciones en la temperatura anual $\left({ }^{\circ} \mathrm{C}\right)$ de 1979 a 2099 para 
las corridas analizadas en la región del PNCh. La mediana de los datos para el escenario rcp4.5 para finales del siglo XXI está alrededor de los $18{ }^{\circ} \mathrm{C}$, mientras que para el escenario rcp8.5 está alrededor de los $20{ }^{\circ} \mathrm{C}$, o sea unos $2{ }^{\circ} \mathrm{C}$ superior al escenario más optimista de los dos. Los gráficos de cajas muestran la dispersión entre las corridas.

La figura 4 es equivalente a la figura 3, pero para las variables precipitación. Cabe resaltar que los cambios observados para el escenario rcp4.5 para finales del siglo XXI son muy pequeños al compararlos con la línea base, mientras que para el escenario rcp8.5 muestra una disminución de menos de $250 \mathrm{~mm}$ en la mediana del acumulado anual al compararlo también con la línea base.

La figura 5 muestra los cambios en temperatura $\left({ }^{\circ} \mathrm{C}\right)$ y en precipitación (\%) para dos horizontes de tiempo en el PNCh, 20392059 y 2079-2099, representados los primeros por los círculos gris y negro y los segundos por los círculos blanco y negro. El escenario corresponde al pesimista en relación a las emisiones de gases de efecto invernadero, es decir, el rcp8.5. Se observó que los cambios con respecto a la línea base en la temperatura para mediados del siglo XXI fueron bastante homogéneos espacialmente, con valores entre 2.5 y $2.6^{\circ} \mathrm{C}$, mientras que para finales del siglo el rango de variación espacial estuvo entre 4.2 y $4.4{ }^{\circ} \mathrm{C}$. Para el caso de la precipitación, la figura 5 muestra los cambios a mitad del siglo XXI fueron muy pequeños mostrando un leve incremento de la precipitación en la vertiente del Caribe y una disminución en la vertiente del Pacífico, sin embargo, por su tamaño se confunden con la variabilidad natural. Hacia finales del siglo, toda la región mostró una disminución de la precipitación que osciló entre $3.7 \%$ (principalmente hacia el oeste de la región) y $5.5 \%$ (principalmente hacia el este del parque). Es natural que haya diferencias entre las vertientes en una región topográfica compleja. Hay pequeñas pero apreciables diferencias entre los resultados de ambas vertientes. No obstante, los resultados de los modelos pueden tener dificultades para mostrar estas diferencias. Casualmente por eso es que lo adecuado es hacer un ajuste de escala como se hizo en este trabajo.

Tal como lo proyectan la mayoría de los modelos globales, el escenario futuro de un clima más seco en todo el país no es del todo consistente y razonable con el conocimiento actual de la variabilidad climática, lo cual pone de manifiesto que efectivamente esos detalles o características regionales no pueden ser bien determinados o simulados por los modelos globales debido a su baja resolución. El modelo estima menos precipitaciones que el clima actual simulado, en porcentajes que oscilan de un $10 \%$ en el Valle Central y Zona Norte, $20 \%$ en el Valle del General.

\section{DISCUSIÓN}

El mapeo de los humedales dentro del PNCh muestra que estos únicamente se encuentran en las laderas de montaña modeladas por acción glaciar, donde actualmente domina la vegetación de páramo. Cabe resaltar que los humedales de tipo lacustre, de mayor tamaño y profundidad, se han podido desarrollar durante los últimos 10 ka posteriores al Último Máximo Glaciar (Orvis \& Horn, 2000) en zonas de antiguo avance glaciar, que han dejado en la actualidad depósitos morrénicos que favorecen esta dinámica acumulativa.

En las zonas de pendiente mayor a $15^{\circ}$, la dinámica de avance y posterior retroceso de los hielos favoreció la formación de superficies rocosas con morfologías de lomeríos, así como gran cantidad de lagunas estacionales. Su reducido tamaño, así como su poca profundidad $\mathrm{y}$ el hecho de estar prácticamente una sobre otra, colabora a que muchos de estos humedales también se sequen rápidamente, tanto por evaporación como por infiltración, generando procesos de recarga-secado que incluso pueden ser diarios, haciéndolos sumamente activos hídricamente, y aumentando aún más su importancia y su vulnerabilidad ante cambios sutiles en estos procesos.

La poca profundidad del suelo en este tipo de humedales, aunado a lo compacto de las 

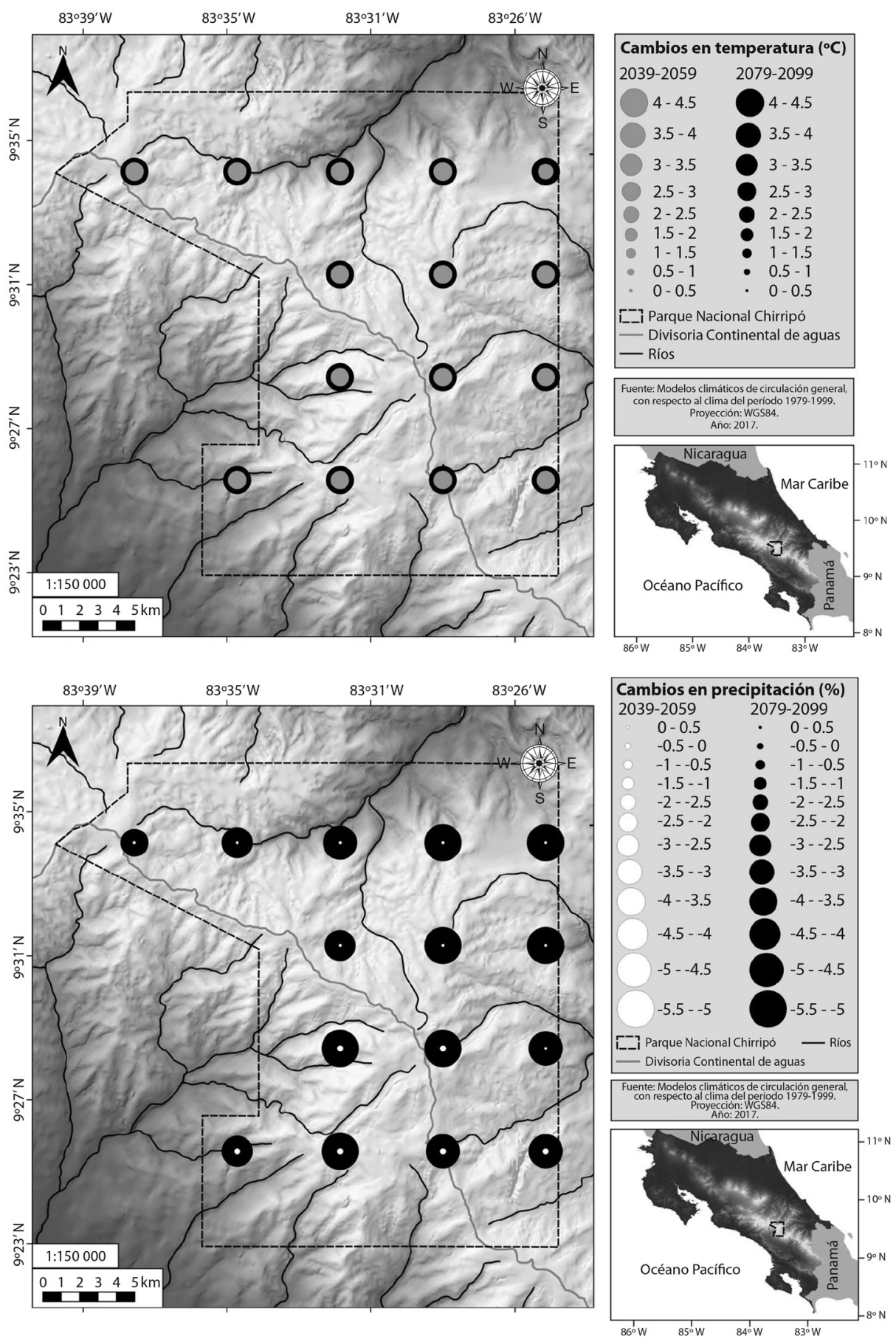

Fig. 5. Cambios en temperatura $\left({ }^{\circ} \mathrm{C}\right)$ y precipitación $(\%)$, para dos horizontes de tiempo en el PNCh. Puntos hacia la izquierda de la divisoria de aguas corresponden a la vertiente del Pacífico y aquellos a la derecha están ubicados en la vertiente del Caribe.

Fig. 5. Changes in temperature $\left({ }^{\circ} \mathrm{C}\right)$ and precipitation $(\%)$, for two time horizons in the PNCh. Locations to the left of the continental divide correspond to the Pacific slope, and locations to the right of the continental divide correspond to the Caribbean slope. 
rocas que se encuentran debajo, hacen que se dé una escorrentía subsuperficial entre el perfil del suelo y la roca madre (Pilgrim, Cordery, \& Baron, 1982). Esta dinámica se potencia por las fuertes pendientes del área, lo cual hace que estas pequeñas depresiones y terrazas de solifluxión (descritas por Lachniet \& Seltzer, 2002) se encuentren generalmente de manera escalonada unas sobre otras, haciendo que el agua migre desde las terrazas superiores a las inferiores. El origen glacial es particularmente importante a la hora de caracterizar los humedales altoandinos en el páramo del $\mathrm{PNCh}$, ya que por su poca profundidad y desarrollo, los suelos en estas zonas no permiten la existencia de pantanos permanentes, como bofedales y turberas, que sí se encuentran en los páramos sudamericanos.

Los escenarios de cambio climático se discuten a partir de un escenario socioeconómico de concentraciones optimista, rcp4.5 y otro pesimista rcp8.5. Los resultados muestran que el escenario rcp4.5 presenta cambios muy pequeños en la región del $\mathrm{PNCh}$ durante el siglo XXI, por lo que si el mundo futuro evoluciona bajo estas condiciones, los impactos para los ecosistemas en esta región serían muy pequeños o inexistentes. Por su parte el correspondiente al rcp8.5 muestra una evolución a través del siglo XXI hacia condiciones más cálidas y secas en toda la región del parque, es decir, un aumento de la temperatura y una disminución de la precipitación. Lo anterior, estaría relacionado con un incremento en el índice de aridez en esa región (Hidalgo, Amador, Alfaro, \& Quesada-Montano, 2013), por lo que los impactos en los ecosistemas deberían abordarse desde un posible impacto de condiciones más áridas, especialmente hacia finales del siglo XXI. El problema real es la rapidez con que ocurren estos cambios, se sugiere que en menos de 100 años los cambios son significativos (especialmente en temperatura), lo cual no permite a algunas especies a adaptarse.

Cabe mencionar que la resolución mensual de los datos climáticos no permite un análisis de los impactos de los eventos extremos a escalas temporales más rápidas (semanas a días o menos) que podrían causar perturbaciones importantes en la ecología de la región aun cuando ocurran de manera poco frecuente.

Respecto a la generación y provisión de agua, bajo los escenarios descritos, la evapotranspiración de la vegetación, el contenido de humedad y materia orgánica de los suelos y los espejos de agua del $\mathrm{PNCh}$ pueden ser afectados por el calentamiento. Estas variables tienen repercusión directa sobre la hidrología de las cuencas que se abastecen desde estos sectores altoandinos (Cuesta, Bustamante, Becerra, Postigo, \& Peraldo, 2012). Al ser el inicio de la fase terrestre del ciclo, disminuyen igualmente los caudales que descienden de manera superficial en ríos y/o quebradas, así como el líquido que se infiltra en el perfil de suelo de las lagunas estacionales, y que recargan acuíferos y manantiales en sectores más bajos.

En el caso de la regulación hídrica, un posible aumento en las temperaturas (Fig. 3), así como en los regímenes de la estacionalidad (Fig. 4 y Fig. 5), implican un impacto directo en los humedales, especialmente en las lagunas estacionales debido a su menor superficie y profundidad. Los lacustres tienen una resiliencia mayor debido a su volumen y que al estar en zonas más bajas, captan más agua que los palustres antes mencionados.

De manera paralela, en el páramo el agua se almacena mayoritariamente en los suelos (Llambí et al., 2012), por lo que estos escenarios podrían repercutir en la capacidad de los mismos para retener y distribuir temporalmente los procesos hidrológicos, que son los que mantienen y liberan los flujos de agua, donde son esenciales como hábitats para las aves durante sus migraciones, y para que las especies animales beban, especialmente en épocas de estiaje (Cuesta et al., 2012), cuando la alta radiación seca la humedad en la superficie.

Si bien el área total de humedales registrada no supera las 50 ha, la identificación y mapeo de más de 950 humedales palustres, nunca antes inventariados de manera oficial, así como la consolidación de información para las lagunas, muestra el vital servicio del ecosistema paramuno como inicio del ciclo terrestre 
del agua, tanto a nivel superficial como subterráneo, confirmando y justificando el acierto al declarar al Chirripó como área silvestre protegida, siendo esta la medida más importante para ayudar a su conservación.

Por tanto, cabe resaltar que los humedales altoandinos no son aislados, sino sistemas o complejos esenciales para dinámicas de microcuencas en las altas montañas, así como de otros sistemas hidrográficos (Convención Ramsar \& Estrategia de Humedales Altoandinos, 2008). Los procesos de provisión de agua y la regulación del ciclo hidrológico requieren de una mayor medición y estudio para poder identificarse y dimensionarse, pero de cumplirse los escenarios propuestos, el impacto sobre los humedales, sus múltiples servicios como la captura de carbono, así como los ecosistemas y personas que de ellos dependen será cada vez mayor. Estos servicios no son ilimitados, por lo que su alteración y degradación conllevaría la reducción y hasta eliminación muchos de ellos, razón por la cual es vital trabajar para no rebasar su umbral crítico, que haría irreversible su deterioro.

\section{AGRADECIMIENTOS}

HH y EA agradecen a los siguientes proyectos de la UCR que le suministraron tiempo y recursos: 805-B7-507, B6-143 (ambos apoyados por CONICIT-MICITT), B7-286 (apoyado por UCREA), B4-227, B0-065, A9-532 (apoyado por CSUCA-ASDI) y B0-810. La figura 5 fue producida por el estudiante de la Universidad de Costa Rica Jorge Daniel García Girón, usando datos proporcionados por HH y EA. NVA y AQR agradecen al Proyecto Humedales y al SINAC-ACLAP por toda la colaboración brindada en el trabajo de campo y procesamiento de la información, así como a Gilberth Dondi, por su acompañamiento y conocimiento en los recorridos por el PNCh. Asimismo, al IMN y al ICE por la información meteorológica brindada que ayudó a la calibración de los escenarios.

\section{RESUMEN}

Los humedales, también llamados altoandinos, han sido poco estudiados y caracterizados en Costa Rica. En este estudio se analizan las características geomorfológicas, climatológicas e hidrológicas de los humedales altoandinos del Parque Nacional Chirripó (PNCh), ubicado al centro-sur de Costa Rica, América Central. Para ello se parte de un levantamiento geomorfológico a escala 1:25000 de formas de relieve particularmente de origen glaciar, el Inventario Nacional de Humedales llevado a cabo por el Proyecto Humedales (PNUD-SINAC-GEF), y la determinación de distintos escenarios de cambio climático para los periodos 2039-2059 y 2079-2099. El objetivo de este trabajo es caracterizar el estado actual de estos ecosistemas, explicar cómo y porqué se forman, así como valorar las posibles implicaciones en el ciclo hidrológico y la provisión de agua de estos reservorios debido a las variaciones del clima a futuro. Los resultados denotan la gran importancia de estos humedales para mantener la dinámica ecológica en el PNCh, así como los servicios de provisión de agua y regulación del ciclo hidrológico en las cuencas que derivan de esta área protegida.

Palabras clave: humedales; geomorfología; climatología; cambio climático; hidrología; América Central.

\section{REFERENCIAS}

Ballantyne, C. (2002). Paraglacial geomorphology. Quaternary Science Reviews, 21, 1935-2017.

Bravo, J., \& Windevoxhel, N. (1997). Manual para la identificación y clasificación de humedales en Costa Rica. San José, Costa Rica: MINAE-UICN-Embajada Real de los Países Bajos.

Chaverri, A. (2008). Historia natural del Parque Nacional Chirripó. Heredia, Costa Rica: Editorial INBio.

Cleef, A., \& Chaverri, A. (1992). Phytogeography of the paramo flora of the Cordillera de Talamanca, Costa Rica. En H. Balsley \& J. Luteyn (Eds.), Paramo: An Andean Ecosystem under Human Influence (pp. 45-60). London, England: Academic Press.

Convención Ramsar y Estrategia de Humedales Altoandinos. (2008). Estrategia Regional para la Conservación y el Uso Sostenible de Humedales Altoandinos. Quito, Ecuador: Gobiernos de Ecuador y Chile, CONDESAN, TNC-Chile.

Cuesta, F., Bustamante, M., Becerra, M. T., Postigo, J., \& Peraldo, M. (2012). Panorama andino sobre cambio climático: Vulnerabilidad y adaptación en los Andes Tropicales. Lima, Perú: CONDESAN-SGCAN. 
Denyer, P., \& Alvarado, G. E. (2007). Mapa geológico de Costa Rica. San José, Costa Rica: Librería Francesa. Escala 1:400 000 .

Gustavsson, M., Kolstrup, E., \& Seijmonsbergen, A. C. (2006). A new symbol-and-GIS based detailed geomorphological mapping system: Renewal of a scientific discipline for understanding landscape development. Geomorphology, 77, 90-111.

Hidalgo, H. G., Amador, J. A., Alfaro, E. J., \& QuesadaMontano, B. (2013). Hydrological Climate Change Projections for Central America. Journal of Hydrology, 495, 94-112.

Hidalgo, H. G., \& Alfaro, E. J. (2015). Skill of CMIP5 climate models in reproducing 20th century basic climate features in Central America. International Journal of Climatology, 35, 3397-3421.

Hidalgo, H. G., Alfaro, E. J., \& Quesada-Montano, B. (2017). Observed (1970-1999) climate variability in Central America using a high-resolution meteorological dataset with implication to climate change studies. Climatic Change, 141, 13-28.

Horn, S. (1990). Timing of deglaciation in the Cordillera de Talamanca, Costa Rica. Climate Research, 1, 81-83.

Horn, S., Orvis, K., \& Haberyan, K. (2005). Limnología de las lagunas glaciales en el páramo del Chirripó, Costa Rica. En M. Kappelle, \& S. Horn (Eds.), Páramos de Costa Rica (pp. 161-181). Heredia, Costa Rica: Editorial Instituto Nacional de Biodiversidad.

Horn, S., \& Haberyan, K. A. (2016). Lakes of Costa Rica. En M. Kappelle (Ed.), Costa Rican Ecosystems (pp 656-682). Chicago: University of Chicago Press.

IMN - Instituto Meteorológico Nacional. (2008). Atlas Climatológico Interactivo. Obtenido de https://www. imn.ac.cr/atlas-climatologico

IMN - Instituto Meteorológico Nacional. (2009). Datos de la estación meteorológica Chirripó. Obtenido de https://www.imn.ac.cr/

Kappelle, M. (2005). Hacia una breve descripción del concepto "páramo". En M. Kapelle, \& S. Horn (Eds.), Páramos de Costa Rica (pp. 29-36). Heredia, Costa Rica: Editorial Instituto Nacional de Biodiversidad.

Kapelle, M., \& Uffelen, J. (2005). Los suelos de los páramos de Costa Rica. En M. Kapelle, \& S. Horn (Eds.), Páramos de Costa Rica (pp. 147-159). Heredia, Costa Rica: Editorial Instituto Nacional de Biodiversidad.
Kappelle, M., \& Horn, S. P. (2016). The Páramo Grasslands of Costa Rica's Highlands. En M. Kappelle (Ed.), Costa Rican Ecosystems (pp. 492-523). Chicago: University of Chicago Press.

Islebe, G., \& Hooghiemstra, H. (1997). Vegetation and climate history of montane Costa Rica since the last glacial. Quaternary Science Reviews, 16, 589-604.

Lachniet, M. S., \& Seltzer, G. O. (2002). Late Quaternary glaciation of Costa Rica. Geological Society of America Bulletin, 114, 547-558.

Llambí, L., Soto, A., Célleri, R., De Bievre, B., Ochoa, B., \& Borja, P. (2012). Ecología, hidrología y suelos de páramos. Quito, Ecuador: Proyecto Páramo Andino.

Maldonado, T., Rutgersson, A., Alfaro, E., Amador, J., \& Claremar, B. (2016). Interannual variability of the midsummer drought in Central America and the connection with sea surface temperatures. Advances in Geosciences, 42, 35-50.

Millenium Assesment. (2005). Ecosystems and Human Well-being. Washington D. C., Estados Unidos: Island Press.

MINAET - Ministerio Nacional de Ambiente, Energía y Telecomunicaciones. Decreto Ejecutivo 35803. Criterios técnicos para la identificación, clasificación y conservación de humedales. La Gaceta, Diario Oficial, Costa Rica, 07 enero, 2010.

Morell, K. D. (2015). Late Miocene to recent plate tectonic history of the southern Central America convergent margin. Geochemistry, Geophysics, Geosystems, 16, $3362-3382$

Orvis, K., \& Horn, S. (2000). Quaternary Glaciers and Climate on Cerro Chirripó, Costa Rica. Quaternary Research, 54, 24-37.

Orvis, K., \& Horn, S. (2005). Los glaciares cuaternarios y el clima del cerro Chirripó, Costa Rica. En M. Kape1le, \& S. Horn (Eds.), Páramos de Costa Rica (pp. 185-213). Heredia, Costa Rica: Editorial Instituto Nacional de Biodiversidad.

Pilgrim, D., Cordery, I., \& Baron, B. (1982). Effects of Catchment Size on Runoff Relationships. Journal of Hidrology, 58, 205-221.

PRIAS-CENAT - Centro Nacional de Alta Tecnología (2005). Fotografias aéreas proyecto CARTA: escala 1:25000. San José, Costa Rica.

Proyecto Humedales (s.f.). Capa de humedales del Área de Conservación La Amistad Pacífico. Sin publicar. 
Capa digital generada por el Proyecto Humedales. SINAC-PNUD-GEF. San José, Costa Rica.

Quesada-Román, A. (2016). Peligros geomorfológicos: inundaciones y procesos de ladera en la cuenca alta del río General (Pérez Zeledón), Costa Rica (Tesis de Maestría). Universidad Nacional Autónoma de México, México.

Quesada-Román, A. (2017). Geomorfología Fluvial e Inundaciones en la Cuenca Alta del Río General, Costa Rica. Anuário do Instituto de Geociências - UFRJ, 40, 278-288.
Secretaría de la Convención Ramsar. (2013). Manual de la Convención de Ramsar: Guía a la Convención sobre los Humedales. Gland, Suiza: Ramsar.

Veas-Ayala, N. (2015). Reseña de los Inventarios Nacionales de Humedales en Costa Rica. (Documento técnico). Heredia, Costa Rica: Proyecto Humedales, SINAC-PNUD-GEF.

World Wildlife Fund (WWF) Colombia. (2005). Los Humedales Altoandinos. Coordinación Editorial: Taller de Comunicaciones WWF. Bogotá, Colombia. 